\title{
Proteins in Outer Membrane Vesicles Produced by Burkholderia cepacia are Responsible for Pro-inflammatory Responses in Epithelial Cells
}

\author{
Se Yeon Kim', Seung II Kim², ${ }^{2,}$ Sung Ho Yun², Minsang Shin ${ }^{1}$, Yoo Chul Lee', Je Chul Lee ${ }^{1 *}$ \\ 'Department of Microbiology, School of Medicine, Kyungpook National University, Daegu 41944, Republic of Korea \\ ${ }^{2}$ Drug \& Disease Target Team, Korea Basic Science Institute, Ochang 28119, Republic of Korea \\ ${ }^{3}$ Department of Bio-Analytical Science, University of Science and Technology (UST), Daejeon 34116, Republic of Korea
}

\author{
Corresponding \\ Je Chul Lee, Professor \\ Department of Microbiology, School of \\ Medicine, Kyungpook National \\ University, 680 Gukchaebosang-ro, \\ Jung-gu, Daegu 41944, Republic of \\ Korea \\ Phone : +82-53-420-4844 \\ Fax : +82-53-427-5664 \\ E-mail : leejc@knu.ac.kr
}

Received : September 2, 2020

Revised : November 9, 2020

Accepted : November 10, 2020

No potential conflict of interest relevant to this article was reported.

Copyright (C) 2020 Journal of Bacteriology and Virology

(C) This is an Open Access article distributed under the terms of the Creative Commons Attribution Non-Commercial License

(http://creativecommons.org/

license/by-nc/3.0/).
Gram-negative bacterial pathogens produce outer membrane vesicles (OMVs) and this secreted cargo plays a role in host-pathogen interactions. OMVs isolated from Burkholderia cepacia induce the cytotoxicity and pro-inflammatory responses both in vitro and in vivo, but OMV components associated with host pathology have not been characterized. This study analyzed the proteomes of OMVs produced by B. cepacia ATCC 25416 and investigated whether proteins in B. cepacia OMVs were responsible for host pathology in vitro. Proteomic analysis revealed that a total of 265 proteins were identified in B. cepacia OMVs. Of the $265 \mathrm{OMV}$ proteins, $179(67.5 \%), 32(12.1 \%), 27(10.2 \%), 17(6.4 \%)$, and 10 (3.8\%) were predicted to be located in the cytoplasm, inner membrane, periplasmic space, outer membrane, and extracellular compartment, respectively. Several putative virulence factors were also identified in B. cepacia OMVs. B. cepacia OMVs slightly induced the cytotoxicity in lung epithelial A549 cells, but there was no difference in cytotoxic activity between intact OMVs and proteinase K-treated OMVs. B. cepacia OMVs stimulated the expression of pro-inflammatory cytokine and chemokine genes in A549 cells, but the expression of these cytokine genes was significantly inhibited in A549 cells incubated with proteinase K-treated OMVs. In conclusion, our results suggest that proteins in $B$. cepacia OMVs are directly responsible for pro-inflammatory responses in lung epithelial cells.

Key Words: Burkholderia cepacia, Outer membrane vesicle, Cytotoxicity, Pro-inflammatory response, Proteomes

\section{INTRODUCTION}

Burkholderia cepacia is a member of $B$. cepacia complex (BCC) that is a group of catalase-positive, lactose non-fermenting, gram-negative bacteria comprised of at least 20 different species, including $B$. cepacia, $B$. multivorans, $B$. cenocepacia, $B$. vietnamiensis, $B$. stabilis, $B$. ambifaria, $B$. dolosa, $B$. anthina, and $B$. pyrrocinia $(1,2)$. B. cenocepacia and $B$. multivorans are the most frequently isolated from the patients with cystic fibrosis belonging to the $\operatorname{BCC}(3,4)$. B. cepacia has also emerged as an important human pathogen, especially in healthcare settings, although this microorganism was first discovered as a causative agent of onion skin rot $(5,6)$. BCC bacteria, including $B$. cepacia, pose little medical risk to healthy people, but the patients with underlying diseases such as chronic granulomatous 
diseases, chronic lung diseases or hematologic malignancies are susceptible to BCC infection $(4,7)$. BCC exhibits a relatively low virulence, but produces several virulence-associated determinants, including elastases, gelatinases, hemolysins, lipases, proteases, and siderophores, have been determined, which may play a role in colonization and infection in the hosts and survival of bacteria in environment (8-10). However, secretomes associated with bacterial pathogenesis have not been fully characterized.

All gram-negative bacteria produce and secrete outer membrane vesicles (OMVs) sized with 20-300 nm (11). OMVs are a secreted cargo that transports toxins, virulence factors, and other bacterial molecules to the host cells and can modulate physiology of host cells $(12,13)$. Gram-negative bacterial pathogens, including Escherichia coli (14), Pseudomonas aeruginosa (15), Acinetobacter baumannii (16), A. nosocomialis (17), Stenotrophomonas maltophilia (18), and BCC (19), secrete OMVs during in vitro culture and the secreted OMVs induce host cell cytotoxicity and pro-inflammatory responses. We recently demonstrated that B. cepacia ATCC 25416 and two clinical isolates secreted OMVs during in vitro culture (20). Moreover, B. cepacia ATCC 25416 produced more OMVs under antibiotic stress conditions such as sub-minimum inhibitory concentrations of ceftazidime, meropenem, and trimethoprim/sulfamethoxazole, than under antibiotic-free conditions. OMVs isolated from B. cepacia cultured under antibiotic stress conditions induced significantly higher pro-inflammatory responses in lung epithelial A549 cells than OMVs from B. cepacia cultured under antibiotic-free conditions. However, bacterial molecules associated with the cytotoxicity or pro-inflammatory responses in $B$. cepacia OMVs have not been determined. The aim of this study was to analyze the proteomes of OMVs produced by $B$. cepacia and investigate their contribution to the induction of host cell cytotoxicity and pro-inflammatory responses in vitro.

\section{MATERIALS AND METHODS}

\section{Bacterial strain and cell culture}

B. cepacia ATCC 25416 was purchased from the American Type Culture Collection (Manassas, VA, USA). Bacteria were cultured in lysogeny (LB) broth with shaking at $37^{\circ} \mathrm{C}$. A549 cells originated from human lung epithelial cells were purchased from the Korean Cell Line Bank (Seoul, Korea). A549 cells were grown in RPMI 1640 medium (HyClone, Logan, UT, USA) supplemented with $10 \%$ heat-inactivated fetal bovine serum (HyClone), $2 \mathrm{mM}$ L-glutamine, and 1,000 U/mL penicillin $\mathrm{G} 37^{\circ} \mathrm{C}$ in a humidified atmosphere with $5 \% \mathrm{CO}_{2}$. Cells were seeded in 6- and 96-well plates for the cytokine gene expression and cell viability assays, respectively.

\section{Isolation of OMVs}

The OMVs of B. cepacia ATCC 25416 were purified from bacterial culture supernatants as previously described $(20,21)$. Bacteria were cultured to 1.5 optical density at $A 600\left(\mathrm{OD}_{600}\right)$ in $1 \mathrm{~L}$ of $\mathrm{LB}$ broth with shaking at $37^{\circ} \mathrm{C}$. Bacterial culture was centrifuged at 8,000 g for $20 \mathrm{~min}$ at $4^{\circ} \mathrm{C}$ and supernatants were filtered using a bottle-top filter with a $0.22 \mu \mathrm{m}$ membrane. The filtered supernatant samples were concentrated using a QuixStand Benchtop System (GE Healthcare, Amersham, UK) with a $500 \mathrm{kDa}$ hollow fiber membrane (GE Healthcare). OMVs were collected by ultracentrifugation at 150,000 $\mathrm{g}$ for $3 \mathrm{~h}$ at $4^{\circ} \mathrm{C}$ and then washed in phosphate-buffered saline (PBS) followed by another ultracentrifugation cycle. OMV pellets were resuspended in 100-200 $\mu \mathrm{l}$ of PBS. The protein concentration of OMVs was determined using a modified BCA assay (Thermo Scientific, Waltham, MA, USA). The purified OMVs were streaked on blood agar plates to check for sterility and then stored at $-80^{\circ} \mathrm{C}$ until use. B. cepacia OMVs were treated with $0.1 \mathrm{\mu g} / \mathrm{ml}$ proteinase $\mathrm{K}$ (Fermentas, St. Leon-Rot, Germany) for $3 \mathrm{~h}$ at $50^{\circ} \mathrm{C}$ for the degradation of OMV proteins (22). 


\section{Protein identification}

Proteins in B. cepacia OMVs were identified using one-dimensional gel electrophoresis and liquid chromatography-tandem mass spectrometry (1-DE-LC-MS/MS) as previously described $(23,24)$. Proteins of OMVs (15 $\mu$ g) were separated on a $12 \%$ sodium dodecyl sulfate-polyacrylamide gel electrophoresis (SDS-PAGE) and divided into eight fractions according to molecular weight. In-gel digestion was performed as previously described (24). Tryptic peptide mixtures were recovered after tryptic digestion, and the peptide extracts were pooled and lyophilized. Peptide samples were then concentrated on a 2G-V/N trap column (Waters, Milford, MA, USA), and concentrated peptides were eluted into a $10 \mathrm{~cm} \times 75 \mu \mathrm{m}$ (i.d.) C18 reversed-phase column at a flow rate of $300 \mathrm{nl} / \mathrm{min}$. High performance liquid chromatography conditions and search parameters for tandem mass spectrometry (MS/MS) analysis were applied. All MS and MS/MS spectra were acquired using an LTQ-Velos ESI ion trap mass spectrometer in data-dependent mode. For protein identification, nano-LC-MS/MS spectra were searched using MASCOT version 2.4 (Matrix Science, UK) with protein sequences obtained from the $B$. cepacia ATCC 25416 genome. A proteomic analysis was performed in triplicate with different samples, and proteins identified in all three experiments were analyzed. Locations of proteins were predicted using the subcellular location prediction program, CELLO version 2.5 (http://cello.life.nctu.edu.tw/). The exponentially modified protein abundance index (emPAl) and mol\% were acquired using MASCOT software (25). Proteins identified in B. cepacia OMVs were classified according to Gene Ontology (GO) functions using Blast2Go Pro software (https://www.blast2go.com/).

\section{Cell viability}

Cell viability was measured using the 3-[4,5-dimethylthiazol-2-yl]-2,5 diphenyltetrazolium bromide (MTT) assay (Abcam, Cambridge, UK). A549 cells were seeded at a concentration of $2.0 \times 10^{4} /$ well in a 96-well microplate. Cells were incubated with either intact B. cepacia OMVs or proteinase K-treated OMVs for $24 \mathrm{~h}$ and then cell viability was measured $2 \mathrm{~h}$ after treatment with MTT reagent at $600 \mathrm{~nm}$.

\section{Quantitative real-time polymerase chain reaction ( $\mathrm{qPCR}$ ) of cytokine genes}

A549 cells cultured in 6-well plates were incubated with 0,10 , and $20 \mu \mathrm{g} / \mathrm{ml}$ of intact $B$. cepacia OMVs or proteinase K-treated OMVs for $6 \mathrm{~h}$. Total RNA was extracted using an RNeasy Mini Kit (Qiagen, Valencia, CA, USA) according to the manufacturer's instructions. CDNA was synthesized by the reverse transcription of $2 \mu \mathrm{g}$ of total RNA using oligo dT primers and TOPscript ${ }^{\mathrm{TM}}$ reverse transcriptase (Enzynomics, Daejeon, Korea) in a total reaction volume of $20 \mu$. qPCR was performed to determine the expression levels of genes encoding glyceraldehyde 3-phosphate dehydrogenase (GAPDH), interleukin (IL) 1B, IL6, IL8, tumor necrosis factor (TNF), and C-C motif chemokine ligand 2 (CCL2) as previously described $(26,27)$. Quantification of the gene transcripts was performed using a StepOnePlus ${ }^{\text {TM }}$ Real-Time PCR System (Applied Biosystems, Foster City, CA, USA) with TOPreal ${ }^{\text {TM }}$ qPCR 2 X PreMIX (SYBR Green with high ROX) (Enzynomics) according to the manufacturer's instructions. The amplification specificity was evaluated using melting curve analysis. Gene expression was normalized to GAPDH expression in each sample, and the fold change was determined using the $\Delta \Delta \mathrm{Ct}$ method. Each experiment was performed in triplicate.

\section{Data analysis and statistics}

Cell death and cytokine gene expression between intact OMVs and proteinase K-treated OMVs were analyzed using Student's t-test. Differences of $P<0.05$ were considered statistically significant. 


\section{RESULTS}

\section{Proteomes of B. cepacia OMVs}

B. cepacia ATCC 25416 was cultured in LB broth and then OMVs were isolated from the culture supernatant. A proteomic analysis of B. cepacia OMVs was performed using 1-DE-LC-MS/MS. A total of 265 proteins were identified in OMVs isolated from B. cepacia ATCC 25416 cultured in LB broth. The most abundant $30 \mathrm{OMV}$ proteins analyzed by emPAI and mol\% were presented in Table 1. Of the 265 proteins, 179 (67.5\%), 32 (12.1\%), 27 (10.2\%), 17 (6.4\%), and 10 (3.8\%) were predicted to be located in the cytoplasm, inner membrane, periplasmic space, outer membrane, and extracellular compartment, respectively (Fig. 1A). A total of 168 proteins identified in the B. cepacia OMVs were classified into 19 groups

Table 1. Major proteins identified in the outer membrane vesicles derived from B. cepacia ATCC 25416 using 1-DE-LC-MS/ MS analysis

\begin{tabular}{|c|c|c|c|c|c|}
\hline No & Protein name & Accession No. & $\mathrm{MW}(\mathrm{Da})$ & Gene ID & Cellular localization \\
\hline 1 & OmpW family protein & WP_027787296.1 & 22,576 & CEQ23_20595 & Outer membrane \\
\hline 2 & Gram-negative porin family protein & WP_027789407.1 & 37,596 & DM41_6493 & Outer membrane \\
\hline 3 & OmpA family protein & WP_021157465.1 & 23,939 & DM41_2287 & Outer membrane \\
\hline 4 & $60 \mathrm{kDa}$ chaperonin & WP_021163332.1 & 57,001 & grol & Cytoplasm \\
\hline 5 & Hypothetical protein & WP_080982090.1 & 10,054 & & Cytoplasm \\
\hline 6 & $30 S$ ribosomal protein $\mathrm{S} 12$ & WP_006400662.1 & 13,991 & rpsL & Cytoplasm \\
\hline 7 & Hypothetical protein & WP_080982195.1 & 7,153 & & Cytoplasm \\
\hline 8 & DUF1289 domain-containing protein & WP_027789832.1 & 7,149 & & Cytoplasm \\
\hline 9 & Hypothetical protein & WP_027790335.1 & 23,396 & CEQ23_37995 & Cytoplasm \\
\hline 10 & Glycine zipper 2TM domain protein & WP_021161893.1 & 15,363 & DM41_1814 & Extracellular \\
\hline 11 & CsbD family protein & WP_021162617.1 & 7,491 & CEQ23_34605 & Cytoplasm \\
\hline 12 & Cold-shock' DNA-binding domain protein & WP_006483056.1 & 7,290 & DM41_6114 & Cytoplasm \\
\hline 13 & Hypothetical protein & WP_081040778.1 & 8,232 & & Cytoplasm \\
\hline 14 & Type-1 fimbrial protein, A chain & WP_027787455.1 & 17,243 & $\operatorname{fim} A$ & Extracellular \\
\hline 15 & Bacterial regulatory s, luxR family protein & WP_057056468.1 & 8,938 & DM41_5655 & Cytoplasm \\
\hline 16 & Flagellin & WP_021161125.1 & 38,736 & DM41_1448 & Extracellular \\
\hline 17 & Hypothetical protein & WP_011352325.1 & 19,742 & DM41_3403 & Extracellular \\
\hline 18 & DUF3562 domain-containing protein & WP_027791570.1 & 9,642 & CEQ23_29815 & Cytoplasm \\
\hline 19 & Hypothetical protein & WP_027792032.1 & 9,617 & DM41_6990 & Cytoplasm \\
\hline 20 & Hypothetical protein & WP_027790331.1 & 20,547 & CEQ23_37970 & Cytoplasm \\
\hline 21 & TPR repeat family protein & WP_027788515.1 & 22,070 & DM41_1686 & Periplasm \\
\hline 22 & DUF883 domain-containing protein & WP_006494044.1 & 10,823 & DM41_2044 & Cytoplasm \\
\hline 23 & Flagellar FliT family protein & WP_027788658.1 & 10,710 & DM41_1446 & Cytoplasmic \\
\hline 24 & Hypothetical protein & WP_027786881.1 & 23,218 & CEQ23_17695 & Periplasm \\
\hline 25 & H-NS histone family protein & WP_021160271.1 & 11,057 & DM41_6992 & Periplasm \\
\hline 26 & Cytochrome c family protein & WP_027790167.1 & 23,519 & DM41_4337 & Cytoplasm \\
\hline 27 & Hypothetical protein & WP_027787567.1 & 11,647 & DM41_2931 & Cytoplasm \\
\hline 28 & Hypothetical protein & WP_027791579.1 & 11,467 & DM41_5965 & Periplasm \\
\hline 29 & Hypothetical protein & WP_063623143.1 & 11,709 & CEQ23_22510 & Periplasm \\
\hline 30 & ATP-dependent protease ATPase subunit HsIU & WP_027788872.1 & 49,850 & $h s / U$ & Cytoplasm \\
\hline
\end{tabular}


based on GO functions; biosynthetic process-associated proteins ( $n=51)$ were the most common category (Fig. 1B). However, 97 proteins could not be classified into a particular functional group, due to poor characterization. Several putative virulence-associated proteins, including phosphocholine-specific phospholipase C (WP_027788973.1), ATP-dependent protease HsIU (WP_027788872.1), ATP-dependent zinc metalloprotease FtsH (WP_021163358.1), and ATP-dependent Clp protease (WP_027786662.1), were identified in OMVs. These results indicate that $B$. cepacia OMVs present a diverse protein profile comprising molecules derived from the cytoplasm, membrane, and extracellular compartment.

(A)

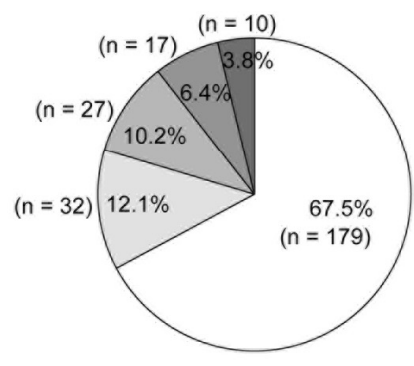

(B)

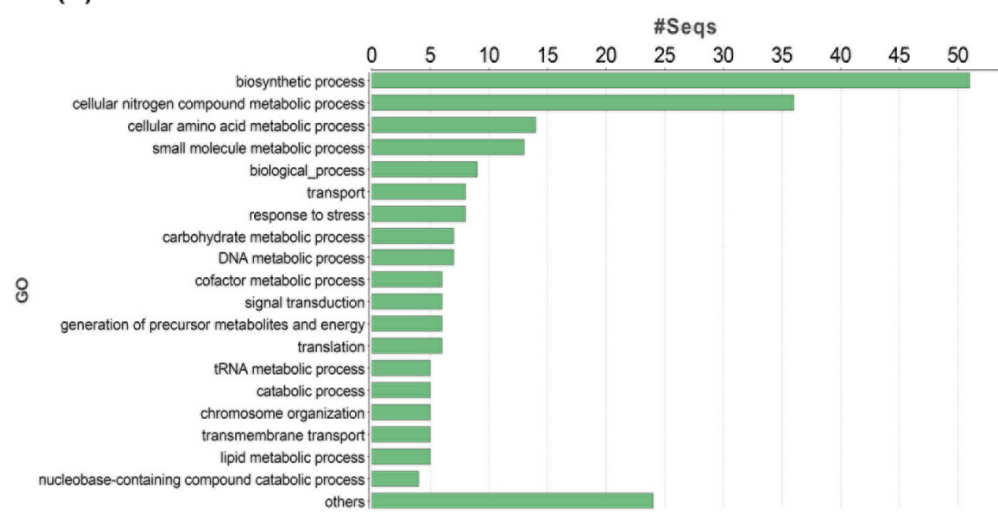

Fig. 1. Proteomic analysis of OMVs isolated from B. cepacia ATCC 25416. Bacterial were cultured in LB broth and OMVs were isolated from culture supernatants. Proteomic analysis was performed using 1-DE-LC-MS/MS. Locations of proteins were predicted using the subcellular location prediction program, CELLO version 2.5 (http://cello.life.nctu.edu.tw/). Proteins identified in B. cepacia OMVs were classified according to Gene Ontology (GO) functions using Blast2Go Pro software (https://www.blast2go.com/). A total of 265 proteins were analyzed by cellular localization (A) and GO (B).

(A)

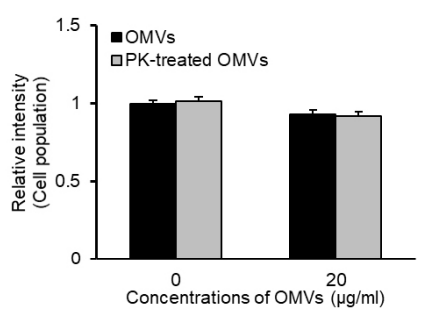

(B)

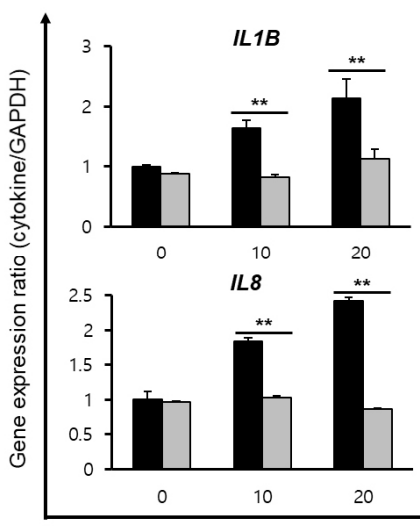

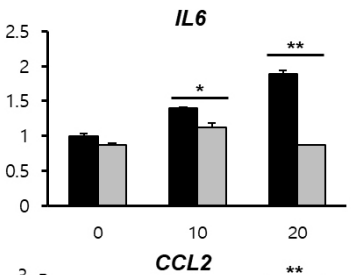

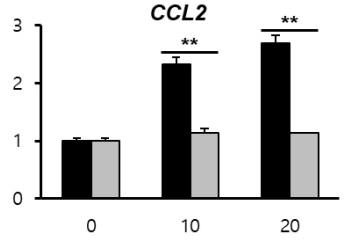

Concentrations of OMVs $(\mu \mathrm{g} / \mathrm{ml})$

Fig. 2. Host cell responses to proteinase $K(P K)$-treated $B$. cepacia OMVs. OMVs were isolated from the culture supernatants of B. cepacia cultured in LB. OMVs were treated with $0.1 \mu \mathrm{g} / \mathrm{mL}$ proteinase $\mathrm{K}$ for $3 \mathrm{~h}$ at $50^{\circ} \mathrm{C}$ for the degradation of $\mathrm{OMV}$ proteins. A549 cells were treated with either intact OMVs or PK-treated OMVs. (A) Cells were treated with $20 \mathrm{\mu g} / \mathrm{mL}$ OMVs for $24 \mathrm{~h}$ and cell viability was determined using MTT assay. Data are presented as mean \pm SD of three independent experiments. (B) Cells were treated with various concentrations of OMVs for $6 \mathrm{~h}$, and gene expression was assessed by qPCR. Data are presented as mean \pm SD of three independent experiments. $* P<0.05, * * P<0.01$ compared to intact OMVs. 


\section{Induction of pro-inflammatory response to proteins derived from B. cepacia OMVs}

We previously showed that OMVs from B. cepacia ATCC 25416 induced host cell cytotoxicity and pro-inflammatory responses in $A 549$ cells in vitro (20). To determine whether proteins in OMVs were directly responsible for cytotoxicity and pro-inflammatory responses, OMVs were treated with proteinase $\mathrm{K}$ and then $\mathrm{A} 549$ cells were incubated with either intact OMVs or proteinase K-treated OMVs. Cell death was not different between intact and proteinase K-treated OMVs (Fig. $2 \mathrm{~A}$ ); however, the proteinase $\mathrm{K}$-treated OMVs did not stimulate the expression of pro-inflammatory cytokine genes $I L 1 B$ and $I L 6$, nor the chemokine genes $I L 8$ and $C C L 2$ (Fig. 2B). The expression of the TNF gene was significantly different between intact and proteinase $\mathrm{K}$-treated $\mathrm{OMVs}$ at $10 \mu \mathrm{g} / \mathrm{ml}$. These results suggest that proteins in $B$. cepacia OMVs are not responsible for host cell cytotoxicity, but are responsible for pro-inflammatory responses in A549 cells.

\section{DISCUSSION}

B. cepacia ATCC 25416 and two clinical isolates, P1311 and P1383, produced OMVs during in vitro culture (20). These $B$. cepacia OMVs induced the host cell cytotoxicity and the expression of pro-inflammatory cytokine and chemokine genes in lung epithelial cells, but specific molecules or components associated with the host cell pathology have not been determined. In the present study, we analyzed the proteomes of OMVs isolated from B. cepacia ATCC 25416 and investigated the contribution of OMV proteins to pro-inflammatory responses in vitro. The present study demonstrated that B. cepacia OMVs contained 256 proteins derived from all bacterial compartments, and that proteins in OMVs were directly responsible for pro-inflammatory responses in vitro.

Gram-negative, non-fermenting bacterial pathogens, including B. pseudomallei (28), B. mallei (29), P. aeruginosa (15), A. baumannii (16), A. nosocomialis (17), and S. maltophilia (18), produced OMVs. OMVs derived from gram-negative, non-fermenting pathogens induced host cell cytotoxicity and pro-inflammatory responses. In addition, $B$. cepacia OMVs induced the cytotoxicity in A549 cells (20), but only $<20 \%$ of cells died at $20 \mu \mathrm{g} / \mathrm{ml}$ protein concentrations of $B$. cepacia OMVs, suggesting that $B$. cepacia OMVs are cytotoxic to host cells. Azurin homologue and hemolysin were found to be cytotoxic among the secretomes produced by $\mathrm{BCC}$ bacteria $(30,31)$, but proteomic analysis revealed that azurin, azurin homologues, and hemolysin were not identified in B. cepacia OMVs in this study. Moreover, there was no significant difference in host cell cytotoxicity between intact OMVs and proteinase K-treated OMVs, indicating that non-protein components in B. cepacia OMVs may be responsible for the host cell cytotoxicity. Instead, the present study identified several putative virulence-associated proteins in $B$. cepacia OMVs. Although $B$. cepacia OMVs are not highly cytotoxic to host cells, it is necessary to clarify cytotoxic factors in $B$. cepacia OMVs for the development of OMV vaccines against $B$. cepacia.

We previously demonstrated that OMVs derived from B. cepacia ATCC 25416 induced the expression of pro-inflammatory cytokine genes, including $\angle L 1 B, I L 6, I L 8, T N F$, and $C C L 2$, in $A 549$ cells, and also induced expression of $I L 1 B, I L 6, C X C L 1 P 1$, $T N F$, and CCL2 genes in the lungs of mice injected with $B$. cepacia OMVs (20). Consistent with the previous study, the present study also showed that OMVs derived from B. cepacia ATCC 25416 induced the expression of pro-inflammatory cytokine and chemokine genes in $A 549$ cells. However, expression of $\angle L 1 B, \angle L 6, I L 8$, and $C C L 2$ genes was completely inhibited in $A 549$ cells incubated with proteinase K-treated B. cepacia OMVs (Fig. 2). These results suggest that proteins packaged in $B$. cepacia OMVs are associated with the expression of these cytokine genes. In the present study, we did not determine the specific proteins associated with pro-inflammatory responses in B. cepacia OMVs. However, our study demonstrates that proteins in $B$. cepacia OMVs are potent pathogen-associated molecular patterns to stimulate pro-inflammatory responses in vitro. The proteomic analysis of OMVs and the contribution of OMV proteins to host cell pathology may improve our understanding of $B$. cepacia pathogenesis. 


\section{ACKNOWLEDGEMENTS}

This work was supported by the National Research Foundation of Korea (NRF) grant funded by the Korea government (NRF-2020R1A2B5B01002228).

\section{CONFLICT OF INTEREST}

Authors declare no conflict of interests in this paper

\section{REFERENCES}

1) Lipuma JJ. Update on the Burkholderia cepacia complex. Curr Opin Pulm Med 2005;11:528-33.

2) Bach E, Sant'Anna FH, Magrich dos Passos JF, Balsanelli E, de Baura VA, Pedrosa FO, et al. Detection of misidentifications of species from the Burkholderia cepacia complex and description of a new member, the soil bacterium Burkholderia catarinensis sp nov. Pathog Dis 2017;75.

3) Kidd TJ, Douglas JM, Bergh HA, Coulter C, Bell SC. Burkholderia cepacia complex epidemiology in persons with cystic fibrosis from Australia and New Zealand. Res Microbio/2008;159:194-9.

4) Kenna DTD, Lilley D, Coward A, Martin K, Perry C, Pike R, et al. Prevalence of Burkholderia species, including members of Burkholderia cepacia complex, among UK cystic and non-cystic fibrosis patients. I Med Microbiol 2017:66:490-501.

5) Burkholder WH. Sour skin, a bacterial rot of onion bulbs. Phytopathology 1950;40:115-7.

6) Abdelfattah R, Al-Jumaah S, Al-Qahtani A, Al-Thawadi S, Barron I, Al-Mofada S, et al. Outbreak of Burkholderia cepacia bacteraemia in a tertiary care centre due to contaminated ultrasound probe gel. J Hosp Infect 2018; 98:289-94.

7) Abdallah M, Abdallah HA, Memish ZA. Burkholderia cepacia complex outbreaks among non-cystic fibrosis patients in the intensive care units: A review of adult and pediatric literature. Infez Med 2018;26:299-307.

8) Tegos GP, Haynes MK, Schweizer HP. Dissecting novel virulent determinants in the Burkholderia cepacia complex. Virulence 2012:3:234-7.

9) Thomson ELS, Dennis JJ. A Burkholderia cepacia complex non-ribosomal peptide- synthesized toxin is hemolytic and required for full virulence. Virulence 2012;3:286-98.

10) Lagatolla C, Skerlavaj S, Dolzani L, Tonin EA, Bragadin CM, Bosco M, et al. Microbiological characterization of Burkholderia cepacia isolates from cystic fibrosis patients: investigation of the exopolysaccharides produced. FEMS Microbiol Lett 2002;209:99-106.

11) Jan AT. Outer membrane vesicles (OMVs) of gram-negative bacteria: a perspective update. Front Microbio/ 2017:8:1053.

12) Kulp A, Kuehn MJ. Biological functions and biogenesis of secreted bacterial outer membrane vesicles. Ann Rev Microbio/2010;64:163-84.

13) Ellis TN, Kuehn MJ. Virulence and immunomodulatory roles of bacterial outer membrane vesicles. Microbio/ Mol Biol Rev2010;74:81-94. 
14) Lee EY, Bang JY, Park GW, Choi DS, Kang JS, Kim HJ, et al. Global proteomic profiling of native outer membrane vesicles derived from Escherichia coli. Proteomics 2007;7:3143-53.

15) Bomberger JM, Maceachran DP, Coutermarsh BA, Ye S, O'Toole GA, Stanton BA. Long-distance delivery of bacterial virulence factors by Pseudomonas aeruginosa outer membrane vesicles. PLoS Pathog 2009;5:e1000382.

16) Jin JS, Kwon SO, Moon DC, Gurung M, Lee JH, Kim SI, et al. Acinetobacter baumannii secretes cytotoxic outer membrane protein A via outer membrane vesicles. PLoS One 2011;6:e17027

17) Nho JS, Jun SH, Oh MH, Park TI, Choi CW, Kim SI, et al. Acinetobacter nosocomialis secretes outer membrane vesicles that induce epithelial cell death and host inflammatory responses. Microb Pathog 2015:81:39-45.

18) Kim YJ, Jeon H, Na SH, Kwon HI, Selasi GN, Nicholas A, et al. Stenotrophomonas maltophilia outer membrane vesicles elicit a potent inflammatory response in vitro and in vivo. Pathog Dis 2016;74:ftw104.

19) Allan ND, Kooi C, Sokol PA, Beveridge TJ. Putative virulence factors are released in association with membrane vesicles from Burkholderia cepacia. Can J Microbio/2003;49:613-24.

20) Kim SY, Kim MH, Son JH, Kim SI, Yun SH, Kim K, et al. Outer membrane vesicles produced by Burkholderia cepacia cultured with subinhibitory concentrations of ceftazidime enhance pro-inflammatory responses. Virulence 2020; 11:995-1005

21) Yun SH, Park EC, Lee SY, Lee H, Choi CW, Yi YS, et al. Antibiotic treatment modulates protein components of cytotoxic outer membrane vesicles of multidrug-resistant clinical strain, Acinetobacter baumannii DU202. Clin Proteomics 2018;15:28.

22) Jeon $\mathrm{H}$, Oh MH, Jun SH, Kim SI, Choi CW, Kwon HI, et al. Variation among Staphylococcus aureus membrane vesicle proteomes affects cytotoxicity of host cells. Microb Pathog 2016:93:185-93.

23) Kwon SO, Gho YS, Lee JC, Kim SI. Proteome analysis of outer membrane vesicles from a clinical Acinetobacter baumannii isolate. FEMS Microbiol Lett 2009;297:150-6.

24) Choi CW, Park EC, Yun SH, Lee SY, Lee YG, Hong Y, et al. Proteomic characterization of the outer membrane vesicle of Pseudomonas putida KT2440. J Proteome Res 2014;13:4298-309.

25) Ishihama Y, Oda Y, Tabata T, Sato T, Nagasu T, Rappsilber J, et al. Exponentially modified protein abundance index (emPAl) for estimation of absolute protein amount in proteomics by the number of sequenced peptides per protein. Mol Cell Proteomics 2005;4:1265-72.

26) Van Faassen H, KuoLee R, Harris G, Zhao X, Conlan JW, Chen W. Neutrophils play an important role in host resistance to respiratory infection with Acinetobacter baumannii in mice. Infect Immun 2007;75:5597-608.

27) Jun SH, Lee JH, Kim BR, Kim SI, Park TI, Lee JC, et al. Acinetobacter baumannï outer membrane vesicles elicit a potent innate immune response via membrane proteins. PLoS One 2013:8:e71751.

28) Nieves W, Petersen H, Judy BM, Blumentritt CA, Russell-Lodrigue K, Roy CJ, et al. A Burkholderia pseudomallei outer membrane vesicle vaccine provides protection against lethal sepsis. Clin Vaccine Immuno/2014;21:747-54.

29) Norris MH, Khan MSR, Chirakul S, Schweizer HP, Tuanyok A. Outer membrane vesicle vaccines from biosafe surrogates prevent acute lethal glanders in mice. Vaccines 2018;6:5.

30) Punj V, Sharma R, Zaborina O, Chakrabarty AM. Energy-generating enzymes of Burkholderia cepacia and their interactions with macrophages. J Bacterio/2003;185:3167-78.

31) Hutchison ML, Poxton IR, Govan JR. Burkholderia cepacia produces a hemolysin that is capable of inducing apoptosis and degranulation of mammalian phagocytes. Infect Immun 1998;66:2033-9. 